\title{
Case Report of Scrub Typhus
}

\author{
SB RABBANI ${ }^{\mathrm{a}}$, MI HOSSAIN ${ }^{\mathrm{b}}$, ABEGUM ${ }^{\mathrm{c}}$
}

\begin{abstract}
Summary:
Scrub typhus is an acute, febrile, infectious illness that is caused by Orientia tsutsugamushi. Scrub and murine endemic typhus infections are under-diagnosed causes of febrile illness across the tropics, and it is not known how common they are in Bangladesh. Here reporting a case who presented with high grade fever for 7 days associated with headache, abdominal discomfort, cough and receiving inj ceftriaxone for 2 days considering as a case of enteric fever. Forty-eight hours after admission, the presence of continued high grade fever, conjunctival congestion, headache, loose motion, cough, low platelet, elevated
\end{abstract}

\section{Introduction :}

Scrub typhus is a mite borne infectious disease distributed throughout the Asia Pacific rim, being endemic in Korea, China, Japan, Bangladesh, Pakistan, India, Thailand, Malaysia, and northern portions of Australia.

Scrub typhus is sometimes misdiagnosed as typhoid fever in tropical countries. Similarly, co-infection of typhoid and typhus fever can be overlooked if not suspected clinically. High grade continued fever with variable associated symptoms, such as malaise, headache, and myalgia, are usually present in both typhoid and typhus fever. Therefore, clinical suspicion is of paramount importance in the diagnosis of both typhoid and typhus fever. Confirmation of the diagnosis is important as the treatment is different

but may not always be possible. In most cases of typhus, treatment is given based on clinical suspicion due to presence of eschar or a positive Weil-Felix test. In

\footnotetext{
a. Dr. Safia Binte Rabbani, Specialist in Medicine, United Hospital Limited.

b. Dr. Md. Iqbal Hossain, Consultant, United Hospital Ltd.

c. Dr. Afsana Begum, Consultant, United Hospital Ltd.

Department of Medicine, United Hospital Limited.
}

Address of Correspondence : Dr. Safia Binte Rabbani, Specialist, Medicine Department, United Hospital Limited. Phone: 01715003919 , Email: safia_rabbani@yahoo.com

Received: 23 Feb. 2017

Accepted: 19 April 2018 transaminase raised the possibility of a different aetiology. The two most likely differentials being dengue and malaria. Finally, typhus was discovered due to history of field visit, presence of eschar and raised titre of WF OXK antigen. All other causes of fever were ruled out by history, clinical examination and relevant investigations. Patient was treated with capsule doxycycline and other supportive therapy and become afebrile within 48 hours. This uncommon clinical scenario should be taken into account in the management of patients with high fever on admission.

(J Bangladesh Coll Phys Surg 2018; 36: 123-127) DOI: http://dx.doi.org/10.3329/jbcps.v36i3.37037

clinically-suspected cases of typhus fever, a rising titre of OX-K antigens supports the diagnosis but confirmation of the diagnosis may be difficult. Isolation of Salmonella Typhi from blood confirms the diagnosis of typhoid fever. Here, we present a case of typhus fever having a eschar and highly rising titre of OXK antigen in weil felix reaction - recently admitted to the United Hospital Limited.

\section{Case Report :}

In August 2016, a previously-healthy 52-year old non smoker, non alcoholic, married, fisheries businessman hailing from Gazipur, admitted with complaints of high grade continued fever for 7 days associated with abdominal discomfort, headache, bodyache, cough. No significant past illness was reported. But for his occupation of fisheries business, he has to visit field regularly and used to do swimming in pond. $\mathrm{He}$ has $\mathrm{H} /$ $\mathrm{O}$ residing in Kala-azar endemic zone but no $\mathrm{H} / \mathrm{O}$ visiting in malaria endemic zone.

On admission, the patient was conscious, oriented, dehydrated with high fever $105^{\circ} \mathrm{F}$. His radial pulse was $140 \mathrm{bpm}$, regular and good in volume; respiration rate was 24 per minute; and BP was 120/80 $\mathrm{mmHg}$. On examination, there were no signs of anemia, jaundice. Skin rash, lymphadenopathy, neck rigidity absent. Systemic examination revealed his breath sounds were vesicular, with no added sounds, abdomen was soft and 
non-tender, no hepato-splenomegali and bowel sound present. First and second heart sounds were normal without any murmur. Neurological and musculoskeletal system examination revealed normal. Relevant investigations were send and intravenous (IV) ceftriaxone 1 gm 12 hourly was started.

Laboratory investigation showed that total white bloodcell count (TWBC) was $8.8 \times 10^{3} / \mu \mathrm{L}$ with polymorphs$76 \%$, lymphocyte-14\%, and monocyte-2\%, platelet: $88 \times 10^{3} / \mu \mathrm{L}$. with raised transaminase. His renal function test,chest X-ray, urine routine examination, USG of whole abdomen were normal. His initial triple antigen test was normal.

After 24 hours, the patient was found to be toxic, hyperthermic and developed conjunctival congestion. After two days, the patient complained of passing loose stool, so a repeat blood count with platelets, serum electrolyte, stool R/E and C/S, ICT for dengue, malaria, Kala-azar were performed. Repeat TWBC was 10,500/ $\mathrm{mm}^{3}$, haematocrit $31 \%$, lymphopenia and platelets $67,000 / \mathrm{mm}^{3}$. Stool examination showed: normal, stool

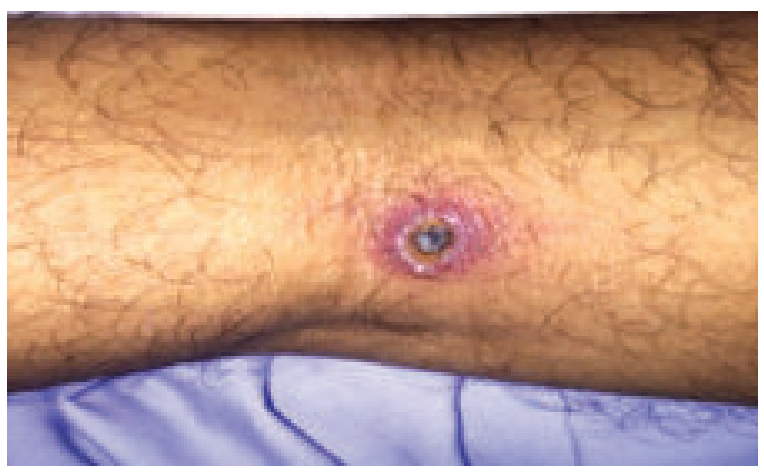

Fig.-1: Eschar in popleteal fossa of patient

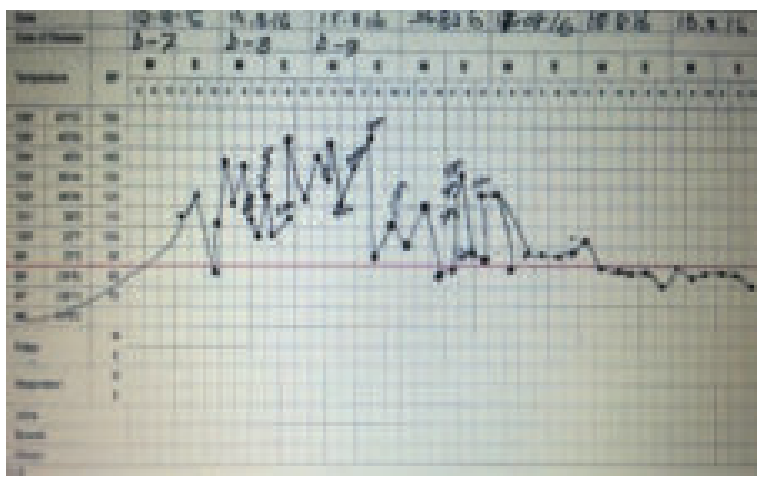

Fig.-2: Temperature chart of patient showing typical remittent fever. After 48 hours of starting doxycycline, fever subsides. for Clostridium difficle toxin : negative, serum electrolytes showed hyponatraemia. Dengue ELISA: Ig M-negative and IgG-negative. PBF for MP : absent, ICT for Malaria and Kala-Azar were negative.

On the $5^{\text {th }}$ day of admission, the patient suddenly develop hypotension not responding to IV fluid challenge and on re-examination of patient there was found to have eschar in right popleteal fossa but no regional lymphadenopathy along with $105^{\circ} \mathrm{F}$ and 70/ 50 mmhg- blood pressure. Blood culture revealed no growth. Widal titres were not rising. 2 nd sample of Weil-Felix titres were: $\mathrm{OX} 2=1: 80, \mathrm{OX} 19=1: 80$, and $\mathrm{OXK}=1: 640$. His hematological report revealed high level of pro calcitonin, raised D- Dimer, FDP and low fibrinogen level. So, patient was diagnosed as a case of septic shock with disseminated intravascular coagulation (DIC) due to scrub typhus and treated with cap doxycycline $100 \mathrm{mg} 12$ hourly and other supportive therapy. After getting 4 dose of doxycycline patient became afebrile, clinically improved and all lab reports came within normal range.

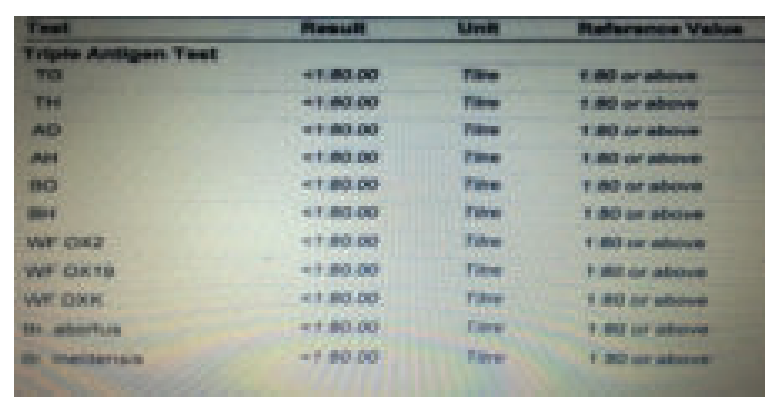

Fig.3: Triple antigen test of patient showing normal titre of WF OXK on 1 st week of fever.

\begin{tabular}{|c|c|c|c|}
\hline Tex & Benut & Uet & Ireferet for \\
\hline \multicolumn{4}{|c|}{ Treve Artigm Tes: } \\
\hline 10 & etsoce & fin & torsatere \\
\hline$T H$ & $\operatorname{cts} x$ & The & foreraleve \\
\hline No & $\operatorname{st} 3000$ & The & t 50 y een \\
\hline$\Delta r$ & $\tan \theta$ & ton & t Nor anow \\
\hline 10 & $\tan \theta$ & hes & 1 Norabien \\
\hline 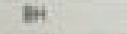 & $\tan \theta$ & $\mathrm{Tm}$ & tenorasos \\
\hline We or & 48000 & the & tols aben \\
\hline WF oxis & tos & for & t $k$ traben \\
\hline Wfors & $t \pm 0 x$ & $\mathrm{~m} n$ & tefs oblow \\
\hline t ands & $\tan x$ & $\mathrm{nen}$ & thereve \\
\hline Ar ralkes & $\operatorname{cts} \theta 0$ & $\mathrm{Pn}$ & t sou abes \\
\hline
\end{tabular}

Fig.-4: Triple antigen test of patient showing raised titre of WF OXK on 2 nd week of fever. 


\section{Discussion:}

Scrub Typhus is a rickettsial infection, which is a group of gram-negative coccobacilli and results an acute febrile illness. Arthropod vectors transfer aetiologic agents to humans. Various types of rickettsial diseases have been described; of them, (a) epidemic/louse-borne typhus is caused by Rickettsia prowazekii, and the vector is the body-louse; (b) Murine typhus is caused by $R$. typhi, and the vector is the rat or cat flea (Xenopsylla cheopis, Ctenocephalides felis). Both epidemic and murine typhus have worldwide distribution; and (c) Scrub typhus is caused by Orientia tsutsugamushi (formerly Rickettsia tsutsugamushi) transmitted via the mite belonging to the Leptotrombidium akamushi, and possibly Leptotrombidium deliense ${ }^{1}$. It is common in Asia, Australia, Papua New Guinea, and Pacific islands. The name derives from the type of vegetation (ie, terrain between woods and clearings) that harbors the vector. Scrub typhus was first described in China in $313 \mathrm{AD}$ and Orientia tsutsugamushi, was first isolated in Japan in 1930. Although scrub typhus was originally recognized as one of the tropical rickettsial diseases, $O$ tsutsugamushi differs from the rickettsiae with respect to cell-wall structure and genetic composition.

The incidence of Scrub typhus is not known. Although most cases are undiagnosed, prospective studies in endemic areas reveal in incidence of 18$23 \% .{ }^{2,3}$ Community surveys in Malaysia reported an incidence of $3.2-3.5 \%$ per month and a seroprevalence exceeding $80 \%$ in those older than 44 years. ${ }^{4}$ Surveillance of military personal deployed in south-east Asia demonstrated seroconversion in 484 per 1000 population. $^{5}$

Geo Sentinal, a global network that monitors travel related morbidity, reported 13 cases of scrub typhus that occurred in travelers from 1996-2011.6 Currently, it is estimated that about 1 million cases of scrub typhus occur annually and that as many as 1 billion people living in endemic areas may have been infected by $O$ tsutsugamushi at some time. ${ }^{7}$

Scrub typhus is often acquired during occupational or agricultural exposures because active rice fields are an important reservoir for transmission. ${ }^{8}$ Humans acquire scrub typhus when an infected chigger bites them while feeding and inoculates $O$ tsutsugamushi pathogens. The bacteria multiply at the inoculation site, and a papule forms that ulcerates and becomes necrotic, evolving into an eschar,with regional lymphadenopathy that may progress to generalized lymphadenopathy within a few days.

Scrub typhus is manifested clinically by high fever $\left(<" 40^{\circ} \mathrm{C}\right)$, intense generalized headache, diffuse myalgias and in many patients, rash and an eschar at the site of the chigger bite. The diagnosis is suggested by the clinical history of exposure, bite by vectors including visit to an endemic area and physical findings of High fever $\left(<" 40{ }^{\circ} \mathrm{C}\right)$, lymphadenopathy, escharpainless papule, hepatomegaly, splenomegaly, and conjunctival congestion are commonly present and confirmed by serologic testing or biopsy of an eschar. Our patient having only high grade remittent fever (Fig2), headache, eschar (fig-1), conjunctival congestion not responding to common antibiotic.

In Bangladesh 40 rickettsial cases were recorded among the admitted febrile patients in $\mathrm{MMCH}$ since 2003 to $2005^{9}$. All 40 cases were presented with fever $(100 \%)$, headache was present in $33(82.5 \%)$ cases, rashes were present in $15(37.5 \%)$ cases, isolated splenomegaly was found in $15(37.5 \%)$ cases \& hepatosplenomegaly in $12(30 \%)$ cases, arthralgia in $13(32.5 \%)$ cases, lymphadenopathy in $5(12.5 \%)$ cases; 2 (5\%) cases attended with unconsciousness \& epistaxis in $1(2.25 \%)$ case. Scrub typhus were 19 (47.5\%), 5 (12.5\%) cases were with dual pathology and were associated with enteric fever. $20(50 \%)$ cases were treated with only doxyclycline \& 5 (12.5\%) cases with tetracycline and ceftriaxone as these cases were associated with enteric fever. All patients (100\%) cured with treatment.

There was another prospective sero-epidemiologic survey was done across six major teaching hospitals in Bangladesh by using an IgM enzyme-linked immunosorbent assay. Results indicated recent exposure (287 of $1,209,23.7 \%$ seropositive for Orientia tsutsugamushi and 805 of $1,209,66.6 \%$ seropositive for Rickettsia typhi). Seropositive rates were different in each region. However, there was no geographic clustering of seropositive results for both organisms. There was no difference between those from rural or urban areas. Rickettsia typhi 
seroreactivity was positively correlated with age. Scrub typhus and murine typhus should be considered as possible causes of infection in Bangladesh. ${ }^{10}$

Routine laboratory studies in patients with scrub typhus reveal early lymphopenia with late lymphocytosis. Thrombocytopenia is also seen. The hematologic manifestations may raise the suspicion of dengue infection ${ }^{11}$ which was consistent with our patient.

Elevated transaminase levels may be present in $75-95 \%$ of patients. Hypoalbuminemia occurs in about $50 \%$ of cases, whereas hyperbilirubinemia is rare. These findings may be especially prevalent in children. In adults, elevated transaminase levels relate to severity of disease. ${ }^{12}$ In our patient all markers of severity were present like hyponatraemia, hypoalbuminaemia and raised transaminase.

The Weil-Felix OX-K strain agglutination reaction can be used to aid in diagnosis of scrub typhus which help in our case also (Fig 4). It may be the only serologic test available in less developed countries; unfortunately, it is not a very sensitive assay. ${ }^{[1]}$

In 2007, Cao et al reported on the development of a rapid diagnostic reagent for scrub typhus. ${ }^{13}$ A study of 2 rapid immune-chromatographic tests for detection of IgM and IgG against $O$. tsutsugamushi determined that both assays were more sensitive and specific than the standard immune-fluorescence assay for the early diagnosis of scrub typhus. ${ }^{14}$ These investigations are not done in our country.

Scrub typhus lasts for 14 to 21 days without treatment. Severe infections may be complicated by interstitial pneumonia, pulmonary edema, congestive heart failure, circulatory collapse, DIC and a wide array of signs and symptoms of central nervous system dysfunction, including delirium, confusion, and seizures. Death may occur as a result of these complications, usually late in the second week of the illness. Our patient develop, circulatory collapse and DIC at the starting of 2nd week of illness when we already started specific treatment on basis of clinical judgments.

By contrast, patients treated with appropriate antibiotics typically become afebrile within 48 hours of starting therapy ${ }^{15,16,17}$. This response to treatment may be useful diagnostically; failure of defervescence within 48 hours is often considered evidence that scrub typhus is not present, and that an alternate diagnosis such as malaria or dengue should be considered. ${ }^{18}$

\section{Conclusion:}

Scrub typhus is emerging as an infectious disease of significant public health problem throughout the globe. Presentation and complications of typhus are widespread. In endemic regions, the infection course making difficult to diagnose due to its similar presentation with other disease like enteric fever and dengue fever. So, in conclusion, patient of scrub typhus treated with doxycycline become afebrile within 48 hours. This response to treatment may be useful diagnostically.

\section{References:}

1. Watt G, Olson JG. Scrub typhus. In : Hunter GW, Thomas SG, eds. Hunters tropical Medicine and Emerging Infectious diseases. 8 th edition. Philadelphia,Pa : WB Sauders and Co; 2000:443-5.

2. Watt G, Paroa P. Scrub typhus and tropical rickettsioses. Curr Opin Infect Dis. Oct 2003;16(5):429-36.

3. Brown GW, Robinson DM, Huxsoll DL, Ng TS, Lim KJ. Scrub typhus: a common cause of illness in indigenous populations. Trans R Soc Trop Med Hyg.1976.70(5-6):444-8.

4. Brown GW, Robinson DM, Huxsoll DL. Serological evidence for a high incidence of transmission of Rickettsia tsutsugamushi in two Orang Asli settlements in Peninsular Malaysia. Am J Trop Med Hyg. 1978 Jan. 27(1 Pt 1):121-3.

5. Corwin A, Soderquist R, Suwanabun N, Sattabongkot J, Martin L, Kelly D, et al. Scrub typhus and military operations in Indochina. Clin Infect Dis. 1999 Oct. 29(4):940-1.

6. Jensenius M, Han PV, Schlagenhauf P, Schwartz E, Parola P, Castelli F, et al. Acute and life threatening tropical diseases in western travelers- A Geo Sentinel multi-centered study,19962011. Am J Trop Med Hyg. Feb 2013; 88(2):393-404.

7. Raoult D. Scrub typhus. Mandell GL, Bennett JE, Dolin R, eds. Mandell, Douglas and Bennett's Principles and Practice of Infectious Diseases. 6th ed. Philadelphia, PA: Elsever Churchill Livingstone; 2005. Vol 2: 2309-10.

8. Sharma PK, Ramakrishnan R, Hutin YJ, et al. Scrub typhus in Darjeeling, India: opportunities for simple, practical prevention measures. Trans R Soc Trop Med Hyg. 2009 Mar 13.

9. Miah MT, Rahman S, Sarker CN, Khan GK, Barman TK. Study on 40 cases of rickettsia. Mymensingh Med J. 2007;16:85-8.

10. Serosurveillance of Orientia tsutsugamushi and Rickettsia typhi in Bangladesh. Maude RR, Maude RJ, Ghose A et al.

11. Watt G, Jongsakul K, Chouriyagune C, Paris R. Differentiating dengue virus infection from scrub typhus in Thai adults with fever. Am J Trop Med Hyg. 2003 May. 68(5):536-8. 
12. Chanta C, Triratanapa K, Ratanasirichup P, Mahaprom W. Hepatic dysfunction in pediatric scrub typhus: role of liver function test in diagnosis and marker of disease severity. $\mathrm{J} \mathrm{Med}$ Assoc Thai. 2007 Nov. 90(11):2366-9.

13. Cao M, Guo H, Tang T, Wang C, Li X, Pan X, et al. Preparation of recombinant antigen of $\mathrm{O}$. tsutsugamushi Ptan strain and development of rapid diagnostic reagent for scrub typhus. $\mathrm{Am}$ J Trop Med Hyg. 2007 Mar. 76(3):553-8.

14. Silpasakorn S, Srisamut N, Ekpo P, Zhang Z, Chao CC, Ching WM, et al. Development of new, broadly reactive, rapid IgG and IgM lateral flow assays for diagnosis of scrub typhus. $\mathrm{Am} \mathrm{J}$ Trop Med Hyg. 2012 Jul. 87(1):148-52.
15. Sheehy TW, Hazlett D, Turk RE. Scrub typhus. A comparison of chloramphenicol and tetracycline in its treatment. Arch Intern Med 1973; $132: 77$

16. Brown GW, Saunders JP, Singh S, et al. Single dose doxycycline therapy for scrub typhus. Trans R Soc Trop Med Hyg 1978; $72: 412$

17. Kim YS, Yun HJ, Shim SK, et al. A comparative trial of a single dose of azithromycin versus doxycycline for the treatment of mild scrub typhus. Clin Infect Dis 2004; 39:1329

18. Lai CH, Huang $\mathrm{CK}$, Weng $\mathrm{HC}$, et al. Clinical characteristics of acute $\mathrm{Q}$ fever, scrub typhus, and murine typhus with delayed defervescence despite doxycycline treatment. Am J Trop Med Hyg 2008; 79:441. 